\title{
SYMPLECTIC MEAN CURVATURE FLOWS IN KÄHLER SURFACES WITH POSITIVE HOLOMORPHIC SECTIONAL CURVATURES
}

\author{
JIAYU LI, LIUQING YANG
}

\begin{abstract}
In this paper, we mainly study the mean curvature flow in Kähler surfaces with positive holomorphic sectional curvatures. We prove that if the ratio of the maximum and the minimum of the holomorphic sectional curvatures is less than 2 , then there exists a positive constant $\delta$ depending on the ratio such that $\cos \alpha \geq \delta$ is preserved along the flow.
\end{abstract}

Mathematics Subject Classification (2000): 53C44 (primary), 53C21 (secondary).

\section{INTRODUCTION}

Mean curvature flows were studied by many authors, for example Huisken ([16], [17]), Ecker-Huisken ([7]), Huisken-Sinestrari [18], Carlo Ilmanen [19], Neves [20], Smoczyk ([21]), Wang ([23]), White [24], etc.

In this paper we mainly concentrated on the symplectic mean curvature flows, which were studied by Chen -Tian [5], Chen-Li [3], Chen-Li-Tian [4], Wang [23], Han-Li [9], [10], [11], Han-Sun [18], Han-Li-Sun [13], and Han-Li-Yang [14]. The basic fact is that the symplectic property is preserved by the mean curvature flow if the ambient space $M$ is Kähler-Einstein, or if the ambient Kähler surface evolves along the Kähler-Ricci flow $[11]$.

Let $(M, J, \bar{\omega}, \bar{g})$ be a Kähler surface. For a compact oriented real surface $\Sigma$ which is smoothly immersed in $M$, the Kähler angle [6] $\alpha$ of $\Sigma$ in $M$ was defined by

$$
\left.\omega\right|_{\Sigma}=\cos \alpha d \mu_{\Sigma}
$$

where $d \mu_{\Sigma}$ is the area element of $\Sigma$ in the induced metric from $\bar{g}$. We say that $\Sigma$ is a symplectic surface if $\cos \alpha>0 ; \Sigma$ is a holomorphic curve if $\cos \alpha \equiv 1$.

Given an immersed $F_{0}: \Sigma \rightarrow M$, we consider a one-parameter family of smooth maps $F_{t}=F(\cdot, t): \Sigma \rightarrow M$ with corresponding images $\Sigma_{t}=F_{t}(\Sigma)$ immersed in $M$ and $F$ satisfies the mean curvature flow equation:

$$
\left\{\begin{array}{l}
\frac{\partial}{\partial t} F(x, t)=H(x, t) \\
F(x, 0)=F_{0}(x)
\end{array}\right.
$$

Key words and phrases. Symplectic mean curvature flow, Kähler angle, Holomorphic sectional curvature, Holomorphic curve. 
where $H(x, t)$ is the mean curvature vector of $\Sigma_{t}$ at $F(x, t)$ in $M$.

Choose an orthonormal basis $\left\{e_{1}, e_{2}, e_{3}, e_{4}\right\}$ on $(M, \bar{g})$ along $\Sigma_{t}$ such that $\left\{e_{1}, e_{2}\right\}$ is the basis of $\Sigma_{t}$ and the symplectic form $\omega_{t}$ takes the form

$$
\omega_{t}=\cos \alpha u_{1} \wedge u_{2}+\cos \alpha u_{3} \wedge u_{4}+\sin \alpha u_{1} \wedge u_{3}-\sin \alpha u_{2} \wedge u_{4},
$$

where $\left\{u_{1}, u_{2}, u_{3}, u_{4}\right\}$ is the dual basis of $\left\{e_{1}, e_{2}, e_{3}, e_{4}\right\}$. Then along the surface $\Sigma_{t}$ the complex structure on $M$ takes the form ([3])

$$
J=\left(\begin{array}{cccc}
0 & \cos \alpha & \sin \alpha & 0 \\
-\cos \alpha & 0 & 0 & -\sin \alpha \\
-\sin \alpha & 0 & 0 & \cos \alpha \\
0 & \sin \alpha & -\cos \alpha & 0
\end{array}\right) .
$$

Recall the evolution equation of the Kähler angle along the mean curvature flow deduced in [11],

Theorem 1.1. The evolution equation for $\cos \alpha$ along $\Sigma_{t}$ is

$$
\left(\frac{\partial}{\partial t}-\Delta\right) \cos \alpha=\left|\bar{\nabla} J_{\Sigma_{t}}\right|^{2} \cos \alpha+\sin ^{2} \alpha \operatorname{Ric}\left(J e_{1}, e_{2}\right)
$$

Here

$$
\left|\bar{\nabla} J_{\Sigma_{t}}\right|^{2}=\left|h_{1 k}^{4}+h_{2 k}^{3}\right|^{2}+\left|h_{2 k}^{4}-h_{1 k}^{3}\right|^{2} \geq \frac{1}{2}|H|^{2} .
$$

We want to see whether the symplectic property is preserved along the mean curvature flow. In the case that $M$ is a Kähler-Einstein surface, we have $\operatorname{Ric}\left(J e_{1}, e_{2}\right)=\bar{\rho} \cos \alpha$, where $\bar{\rho}$ is the scalar curvature of $M$, so the symplectic property is preserved. If the ambient Kähler surface evolves along the Kähler-Ricci flow, Han-Li [11] derived the evolution equation for $\cos \alpha$ and consequently they showed that the symplectic property is also preserved. In this paper, we find another condition to assure that along the flow, at each time the surface is symplectic. Note that we don't require $M$ to be Einstein. Denote the minimum and maximum of holomorphic sectional curvatures of $M$ by $k_{1}$ and $k_{2}$. We state our main theorem as follows:

Main Theorem Suppose $M$ is a Kähler surface with positive holomorphic sectional curvatures. Set $\lambda=\frac{k_{2}}{k_{1}}$. If the flow satisfies either

I. $1 \leq \lambda<\frac{11}{7}$ and $\cos \alpha(\cdot, 0) \geq \delta>\frac{53(\lambda-1)}{\sqrt{(53 \lambda-53)^{2}+(48-24 \lambda)^{2}}}$,

or

II. $\frac{11}{7} \leq \lambda<2$ and $\cos \alpha(\cdot, 0) \geq \delta>\frac{8 \lambda-5}{\sqrt{(8 \lambda-5)^{2}+(12-6 \lambda)^{2}}}$,

then along the flow

$$
\left(\frac{\partial}{\partial t}-\Delta\right) \cos \alpha \geq\left|\bar{\nabla} J_{\Sigma_{t}}\right|^{2} \cos \alpha+C \sin ^{2} \alpha
$$

where $C$ is a positive constant depending only on $k_{1}, k_{2}$ and $\delta$. As a corollary, $\min _{\Sigma_{t}} \cos \alpha$ is increasing with respect to $t$. In particular, at each time $t, \Sigma_{t}$ is symplectic. Therefore, we call this flow the symplectic mean curvature flow.

Since we obtain (1.6), many theorems in "symplectic mean curvature flows in Kähler-Einstein surfaces" still hold in our case. For example, 
Arguing as in [6] by strong maximum principle, we have

Corollary 1.2. I. Suppose $M$ is a Kähler surface with positive holomorphic sectional curvatures and $1 \leq \lambda<\frac{11}{7}$, then every symplectic minimal surface satisfying

$$
\cos \alpha>\frac{53(\lambda-1)}{\sqrt{(53 \lambda-53)^{2}+(48-24 \lambda)^{2}}}
$$

in $M$ is a holomorphic curve.

II. Suppose $M$ is a Kähler surface with positive holomorphic sectional curvatures and $\frac{11}{7} \leq \lambda<2$, then every symplectic minimal surface satisfying

$$
\cos \alpha>\frac{8 \lambda-5}{\sqrt{(8 \lambda-5)^{2}+(12-6 \lambda)^{2}}}
$$

in $M$ is a holomorphic curve.

Arguing exactly in the same way as in [3] or [23], we have

Theorem 1.3. Under the same condition of the Main Theorem, the symplectic mean curvature flow has no type I singularity at any $T>0$.

Acknowledgment: The research was supported by NSFC 11071236, 11131007 and 10421101.

\section{Curvature Tensor, Sectional Curvature and Holomorphic SECTiOnal CuRvature}

Denote the curvature tensor of $M$ by $K$. Set $K(X)=K(X, J X, X, J X)$ and $K(X, Y)=K(X, Y, X, Y)$, where $X, Y$ are arbitrary vector fields on $M$. It is known that (c.f. [1], [22]) we can express the sectional curvatures by holomorphic sectional curvatures.

Theorem 2.1. The sectional curvatures of $M$ can be determined by the holomorphic sectional curvatures by

$$
\begin{aligned}
K(X, Y)= & \frac{1}{32}[3 K(X+J Y)+3 K(X-J Y)-K(X+Y)-K(X-Y) \\
& -4 K(X)-4 K(Y)] .
\end{aligned}
$$

Using (2.1), it is easy to check that,

Theorem 2.2. For any vector fields $X, Y$ and $Z$ on $M$,

$$
\begin{aligned}
& K(X, Y, X, Z) \\
= & \frac{1}{2}[K(Y+Z, X)-K(X, Y)-K(X, Z)] \\
= & \frac{1}{64}[3 K(Y+Z+J X)+3 K(Y+Z-J X)-K(Y+Z+X)-K(Y+Z-X) \\
& -3 K(Y+J X)-3 K(Y-J X)-3 K(Z+J X)-3 K(Z-J X) \\
& -4 K(Y+Z)+K(Y+X)+K(Y-X)+K(Z+X)+K(Z-X)
\end{aligned}
$$




$$
+4 K(X)+4 K(Y)+4 K(Z)]
$$

Denote the minimum and the maximum of sectional curvatures by $K_{\min }$ and $K_{\max }$ respectively, we have the following estimates.

Theorem 2.3. $K_{\min }$ and $K_{\max }$ satisfy

$$
K_{\max } \leq \frac{3}{2} k_{2}-\frac{1}{2} k_{1}
$$

and

$$
K_{\min } \geq \frac{3}{4} k_{1}-\frac{1}{2} k_{2}
$$

Proof. Given any point $p \in M$ and any two unit orthogonal vectors $X$ and $Y$ at $p$, we can find two vectors $Z$ and $W$ such that $\{X, Y, Z, W\}$ form an orthonormal basis of $T_{p} M$. Suppose $J X=y Y+z Z+w W$, then

$$
\langle X+J Y, X+J Y\rangle=2-2 y,
$$

and

$$
\langle X-J Y, X-J Y\rangle=2+2 y .
$$

Assume the Kähler form is anti-self-dual, it was shown in [14] that, $y^{2}+z^{2}+w^{2}=1$ and $J$ has the form

$$
J=\left(\begin{array}{clcr}
0 & y & z & w \\
-y & 0 & w & -z \\
-z & -w & 0 & y \\
-w & z & -y & 0
\end{array}\right)
$$

Combining (2.1) with (2.5) and (2.6), we get

$$
\begin{aligned}
K(X, Y) & \leq \frac{1}{32}\left[3(2-2 y)^{2} k_{2}+3(2+2 y)^{2} k_{2}-2^{2} k_{1}-2^{2} k_{1}-4 k_{1}-4 k_{1}\right] \\
& =\frac{1}{4}\left[\left(3+3 y^{2}\right) k_{2}-2 k_{1}\right] \\
& \leq \frac{1}{4}\left(6 k_{2}-2 k_{1}\right) \\
& =\frac{3}{2} k_{2}-\frac{1}{2} k_{1},
\end{aligned}
$$

and similarly

$$
\begin{aligned}
K(X, Y) & \geq \frac{1}{4}\left[\left(3+3 y^{2}\right) k_{1}-2 k_{2}\right] \\
& \geq \frac{1}{4}\left(3 k_{1}-2 k_{2}\right) \\
& =\frac{3}{4} k_{1}-\frac{1}{2} k_{2} .
\end{aligned}
$$

This proves the theorem. 


\section{Proof of the Main Theorem}

In this section, we will prove the Main Theorem of this paper.

Proof of the Main Theorem. In order to prove this theorem, we need to estimate $\operatorname{Ric}\left(J e_{1}, e_{2}\right)$. Using two different methods, we get two available estimates. We now deduce the first one.

$$
\begin{aligned}
\operatorname{Ric}\left(J e_{1}, e_{2}\right)= & K\left(J e_{1}, e_{1}, e_{2}, e_{1}\right)+K\left(J e_{1}, e_{3}, e_{2}, e_{3}\right)+K\left(J e_{1}, e_{4}, e_{2}, e_{4}\right) \\
= & K\left(\cos \alpha e_{2}+\sin \alpha e_{3}, e_{1}, e_{2}, e_{1}\right)+K\left(\cos \alpha e_{2}+\sin \alpha e_{3}, e_{3}, e_{2}, e_{3}\right) \\
& +K\left(\cos \alpha e_{2}+\sin \alpha e_{3}, e_{4}, e_{2}, e_{4}\right) \\
= & \cos \alpha R_{22}+\sin \alpha\left(K_{3121}+K_{3424}\right),
\end{aligned}
$$

where

$$
R_{22}=K_{2121}+K_{2323}+K_{2424} .
$$

By (2.1), we have

$$
\begin{aligned}
K_{2121}= & \frac{1}{32}\left[3 K\left(e_{1}+J e_{2}\right)+3 K\left(e_{1}-J e_{2}\right)-K\left(e_{1}+e_{2}\right)-K\left(e_{1}-e_{2}\right)\right. \\
& \left.-4 K\left(e_{1}\right)-4 K\left(e_{2}\right)\right] .
\end{aligned}
$$

By our choice of the complex structure (1.3), we get

$$
\left\langle e_{1}+J e_{2}, e_{1}+J e_{2}\right\rangle=2-2 \cos \alpha,
$$

and

$$
\left\langle e_{1}-J e_{2}, e_{1}-J e_{2}\right\rangle=2+2 \cos \alpha .
$$

Hence $K_{2121}$ can be estimated by $k_{1}$ and $k_{2}$,

$$
\begin{aligned}
K_{2121} & \geq \frac{1}{32}\left[3(2-2 \cos \alpha)^{2} k_{1}+3(2+2 \cos \alpha)^{2} k_{1}-2^{2} k_{2}-2^{2} k_{2}-4 k_{2}-4 k_{2}\right] \\
& =\frac{1}{4}\left[\left(3+3 \cos ^{2} \alpha\right) k_{1}-2 k_{2}\right] .
\end{aligned}
$$

Similarly, we get

$$
K_{2323} \geq \frac{1}{4}\left(3 k_{1}-2 k_{2}\right)
$$

and

$$
K_{2424} \geq \frac{1}{4}\left[\left(3+3 \sin ^{2} \alpha\right) k_{1}-2 k_{2}\right] .
$$

Putting (3.3), (3.4) and (3.5) into (3.2), we obtain that

$$
R_{22} \geq 3 k_{1}-\frac{3}{2} k_{2}
$$

Using (2.2) and (1.3), we can also estimate $K_{3121}$ and $K_{3424}$. We have

$$
K_{3121} \geq \frac{1}{32}\left[(53+48 \sin \alpha \cos \alpha) k_{1}-53 k_{2}\right],
$$


and

$$
K_{3424} \geq \frac{1}{32}\left[(53-48 \sin \alpha \cos \alpha) k_{1}-53 k_{2}\right] .
$$

Adding (3.7) and (3.8) yields

$$
K_{3121}+K_{3424} \geq-\frac{53}{16}\left(k_{2}-k_{1}\right) .
$$

By a similar computation in the opposite direction, we get

$$
\left|K_{3121}+K_{3424}\right| \leq \frac{53}{16}\left(k_{2}-k_{1}\right) .
$$

Therefore by (3.1), (3.6), (3.10) and short time existence of the mean curvature flow, we have

$$
\begin{aligned}
\operatorname{Ric}\left(J e_{1}, e_{2}\right) & \geq \cos \alpha\left(3 k_{1}-\frac{3}{2} k_{2}\right)-\sqrt{1-\cos ^{2} \alpha} \frac{53}{16}\left(k_{2}-k_{1}\right) \\
& =\left(3 \cos \alpha+\frac{53}{16} \sqrt{1-\cos ^{2} \alpha}\right) k_{1}-\left(\frac{3}{2} \cos \alpha+\frac{53}{16} \sqrt{1-\cos ^{2} \alpha}\right) k_{2} .
\end{aligned}
$$

If $1 \leq \lambda<2$ and $\cos \alpha>\frac{53(\lambda-1)}{\sqrt{(53 \lambda-53)^{2}+(48-24 \lambda)^{2}}}$, then the RHS of (3.11) is positive.

Another estimate follows directly from Theorem 2.3 and Bergers inequality (c.f. [8]) that

$$
\left|K_{3121}+K_{3424}\right| \leq\left|K_{3121}\right|+\left|K_{3424}\right| \leq K_{\max }-K_{\min } \leq 2 k_{2}-\frac{5}{4} k_{1} .
$$

Putting the above estimate into (3.1) yields

$$
\begin{aligned}
\operatorname{Ric}\left(J e_{1}, e_{2}\right) & \geq \cos \alpha\left(3 k_{1}-\frac{3}{2} k_{2}\right)-\sqrt{1-\cos ^{2} \alpha}\left(2 k_{2}-\frac{5}{4} k_{1}\right) \\
& =\left(3 \cos \alpha+\frac{5}{4} \sqrt{1-\cos ^{2} \alpha}\right) k_{1}-\left(\frac{3}{2} \cos \alpha+\frac{5}{4} \sqrt{1-\cos ^{2} \alpha}\right) k_{2} .
\end{aligned}
$$

It follows that if $1 \leq \lambda<2$ and $\cos \alpha>\frac{8 \lambda-5}{\sqrt{(8 \lambda-5)^{2}+(12-6 \lambda)^{2}}}$, then the RHS of (3.11) is positive. Note that

$$
\frac{53(\lambda-1)}{\sqrt{(53 \lambda-53)^{2}+(48-24 \lambda)^{2}}} \leq \frac{8 \lambda-5}{\sqrt{(8 \lambda-5)^{2}+(12-6 \lambda)^{2}}}
$$

for $1 \leq \lambda<\frac{11}{7}$, and

$$
\frac{53(\lambda-1)}{\sqrt{(53 \lambda-53)^{2}+(48-24 \lambda)^{2}}} \geq \frac{8 \lambda-5}{\sqrt{(8 \lambda-5)^{2}+(12-6 \lambda)^{2}}}
$$

for $\frac{11}{7} \leq \lambda<2$, we get the conclusion.

Q.E.D. 


\section{REFERENCES}

[1] M. Brozos-Vzquez, E. Garca-Ro and P. Gilkey, Relating the curvature tensor and the complex Jacobi operator of an almost Hermitian manifold, Adv. Geom., 8 (2008), no. 3, 353-365.

[2] J. Cheeger and D. Ebin, Comparison Theorems in Riemannian Geometry, American Mathematical Society (1975).

[3] J. Chen and J. Li, Mean curvature flow of surfaces in 4-manifolds, Adv. Math., 163 (2001), 287-309.

[4] J. Chen, J. Li and G. Tian, Two-Dimensional graphs moving by mean curvature flow, Acta. Math. Sinica, English Series, 18 (2002), 209-224.

[5] J. Chen and G. Tian, Moving symplectic curves in Kähler-Einstein surfaces, Acta Math. Sinica, English Series, 16(2000), 541-548.

[6] S. S. Chern and J. Wolfson, Minimal surfaces by moving frams, Amer. J. Math., 105 (1983), 59-83.

[7] K. Ecker and G. Huisken, Mean curvature evolution of entire graphs, Ann. of Math., 130 (1989), 453-471.

[8] S. Goldberg, Curvature and Homology, Academic Press, London, 1962.

[9] X. Han and J. Li, The mean curvature flow approach to the symplectic isotopy problem, IMRN, 26 (2005), 1611-1620.

[10] X. Han, J. Li, Translating solitons to symplectic and Lagrangian mean curvature flows, Internat. J. Math. 20(4) (2009), 443C458.

[11] X. Han and J. Li, The mean curvature flow along the Kähler-Ricci flow, arXiv: math.DG/.1105.1200v1.

[12] X. Han and J. Li, Simplectic critical surfaces in Kähler surfaces, J.Eur.Math.Soc.,12(2) (2010), 505-527.

[13] X. Han, J. Li and J. Sun, The second type singularities of symplectic and Lagrangian mean curvature flows, Chin. Ann. Math. Ser. B 32 (2011), no. 2, 223-240.

[14] X. Han,J. Li and L. Yang, symplectic mean curvature flow in $\boldsymbol{C} P^{2}$, to appear in Cal. Var. PDE.

[15] X. Han, J. Sun, Translating solitons to symplectic mean curvature flows, Ann. Glob. Anal. Geom., 38 (2010), 161-169.

[16] G. Huisken, Flow by mean curvature of convex surfaces into spheres, J. Diff. Geom., 20(1984), 237-266.

[17] G. Huisken, Asymptotic behavior for singularities of the mean curvature flow, J. Diff. Geom. 31 (1990),

[18] G. Huisken and C. Sinestrari, Mean curvature flow with surgeries of two-convex hypersurfaces, Invent. Math. 175 (2009), 137C221.

[19] T. Ilmanen, Elliptic Regularization and Partial Regularity for Motion by Mean Curvature, Mem. Am. Math. Soc., 520 (1994).

[20] A. Neves, Singularities of Lagrangian mean curvature flow: zero-Maslov class case, Invent. Math. 168(3) (2007), 449C484.

[21] K. Smoczyk, Der Lagrangesche mittlere Kruemmungsfluss, Univ. Leipzig (Habil.-Schr.), 102 S. 2000.

[22] L. Vanhecke, Some almost Hermitian manifolds with constant holomorphic sectional curvature, J. Diff. Geom., 12 (1977), no. 4, 461-471.

[23] M.-T. Wang, Mean curvature flow of surfaces in Einstein four manifolds, J. Diff. Geom., 57 (2001), 301-338.

[24] B. White, A local regularity theoreom for mean curvature flow, Ann. Math. 161 2005, 14871519 . 
Jiayu Li, School of Mathematical Sciences, University of Science and Technology of China Hefei 230026, AMSS CAS Beijing 100190, P. R. China

E-mail address: lijia@amss.ac.cn

Liuqing Yang, Academy of Mathematics and Systems Sciences, Chinese Academy of Sciences, BeiJing 100190, P. R. of China.

E-mail address: yangliuqing@amss.ac.cn 